\title{
Flash-induced stabilized images
}

\author{
HENRY J. MICHALEWSKI \\ California State College at Long Beach. Long Beach, Calif. 90801
}

Six Ss observed the afterimages of 11 flash-produced stimulus targets; they reported fading, regeneration, and image breakdown similar to results reported for stabilization studies employing contact lenses and afterimage techniques.

Visual targets viewed as stabilized images produce at least two major phenomena: (1) fading of the stimulus target and (2) regeneration and/or disappearance of portions of the target. Explanations for (1) and (2) at both physiological and psychological levels are reportedly centered on variations in receptor stimulation and the characteristics of the stimulus object, including meaning, patterning, proximity, complexity, and physical size (Ditchburn \& Pritchard, 1952; Ditchburn \& Fender, 1955; Evans \& Piggins, 1963; Hebb, 1963; Pritchard, Heron, \& Hebb, 1960; Pritchard, 1961; Ratliff, 1952; Riggs, Ratliff, Cornsweet, \& Cornsweet, 1953). Fragmentation phenomena are also reported for luminous objects (Eagle, Bowling, \& Klein, 1966; McKinney, 1963; Minard \& Batcher, 1967; Schuck, Brock, \& Becker, 1964).

Many of the above studies achieved stabilization by means of some type of optical projection system attached directly to the eye. That some of the fragmentation and regeneration of stabilized images may be due to imperfections in the method of stabilization was discussed by Barlow (1963). Heckenmueller (1965) reviewed stabilization methods and mentioned some of the controversy over the various techniques. Yarbus (1967) developed a tightly fitting eye cap which he maintained removed slippage and resulted in stabilized images that faded and disappeared without regeneration.

Bennet-Clark \& Evans (1963) introduced an alternative procedure for producing stabilized images without attachments to the eve. By presenting the visual objects to be siudied via a bright flash, the resulting afterimage can be viewed as a perfectly stabilized retinal image. In this study and later ones (Evans \& Marsden, 1966; Evans, 1967), fragmentation, regeneration, and disappearance of afterimages closely resembled the findings of corneal stabilization methods. Barlow \& Sparrock (1964) also reported the similarity of fading afterimages and corneal stabilization

*The writer wishes to acknowledge the helpful suggestions made by Doris $\mathrm{C}$. DeHardt and the cooperation extended by Reed S. Boswell. for a spot of light. The purpose of the present study was to select representative stimulus targets from previous corneal studies (Pritchard et al, 1960, 1961) for presentation via a momentary flash; disappearance, fading, and image breakdown phenomena can then be compared with other stabilization findings.

\section{SUBJECTS}

Six adults, four male and two female, working at the Long Beach Veterans' Hospital served as Ss.

\section{APPARATUS}

A slide viewer with light baffles and diffusing screen was constructed to hold the flash cube and slides in proper alignment. Appropriate internal and external parts of the viewer were painted dull flat black. The viewer stand could be positioned to adjust conveniently the angle and distance of the stimulus target from the $S$. Figure 1 pictures the essential elements of the viewer in cutaway section. STIMULUS MATERIALS

Eleven stimulus targets were drawn and photographed. The film was successively processed to produce high-contrast negative transparencies; the negatives were mounted in standard $2 \times 2$ in. glass slide carriers. When viewed at a distance of $500 \mathrm{~mm}$, the targets subtended a visual field of not more than $2 \mathrm{deg}$ and a line thickness of $5 \mathrm{~min}$ of arc (except for Stimuli 1 and 3 , which subtended 2 and $7 \mathrm{~min}$ of arc, respectively). The 11 targets and the practice slide, a clear disk, appear in Fig. 2 in positive form.

\section{PROCEDURE}

Each $S$ was comfortably seated in front of the viewer and brought into final alignment with the stimulus target by being instructed to center his gaze in the viewer chamber by the reflection of his eye from the glass slide carrier. The targets were presented to the right eye of the $S$ (at a distance of $500 \mathrm{~mm}$ ), with the left eye temporarily occluded by a card during the flash presentation. At the "okay" of the S, the flash was delivered; he then looked up from the viewer, straight ahead, for the appearance of the afterimage on a white card $5 \mathrm{ft}$ away. The afterimage was observed for a 2-min trial period with a 6-min rest period before the next stimulus presentation. Each of the Ss was presented

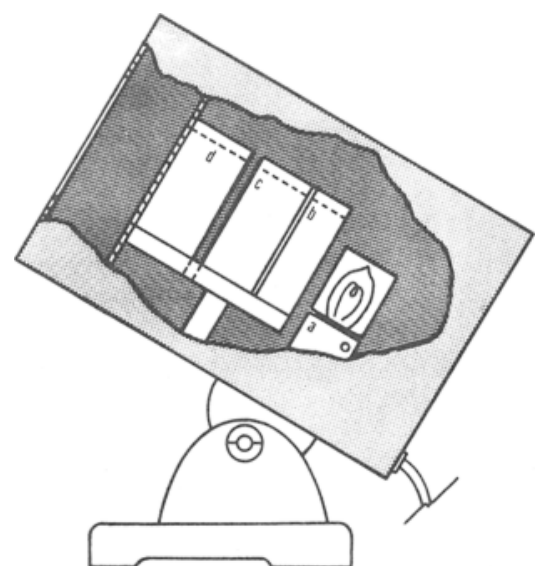

Fig. 1. A cutaway section of the viewer showing (a) the flash-cube unit and flash cube, (b) diffusing screen, (c) slot for individual slides, and (d) the viewing chamber.

four stimulus targets in random order on each of three consecutive days; the practice slide, however, was presented only once on the first day to familiarize the $S$ with viewing afterimages. All trials were conducted in a cubicle where the luminance of the test card was measured at $30 \mathrm{fL}$.

Verbal reports of image transformations and two time measures were taken. The $S$ was given the option of reporting the nature of image breakdown and image transformations during the trial or afterwards, either verbally and/or by drawings. The time measures recorded were (1) latency, the period from flash to the first appearance of the afterimage and (2) the total cumulative time the afterimage was seen in geometrical entirety. The $S$ was provided with a lever switch connected to Hunter timers and was

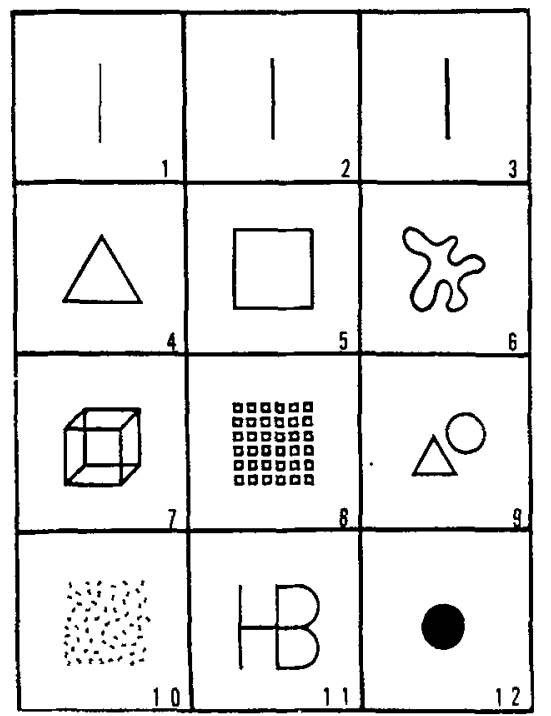

Fig. 2. The 11 stimulus targets and practice slide shown in positive form. 

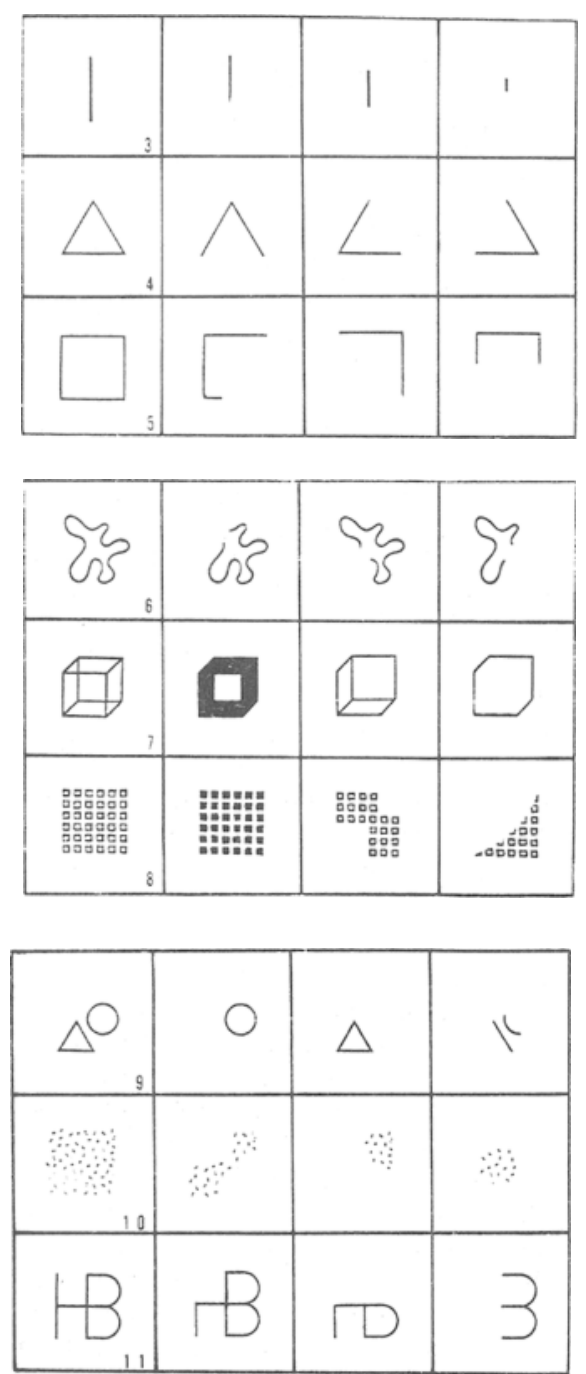

Fig. 3. (a,b.c) The stimulus targets and some image breakdown transformations for various fading periods.

given instructions to press the lever at the instant the original afterimage test target was seen, for the duration of the intact afterimage and every time thereafter. The $S$ was told that the afterimage might undergo color changes but that whatever the color of the target, whenever it appeared in geometrical form as originally presented, the lever was to be pressed.

\section{RESULTS}

All targets underwent fading, regeneration, and image breakdown. For all Ss a typical trial period consisted of fading, breakdown, and regeneration of the complete geometrical image. Figure 3 presents some of the transformations reported by Ss for Stimuli 3-11 (1 and 2 were similar to 3). The transformations are not necessarily sequentially depicted (e.g., $4-8,10$, and 11) but also represent stages after the image reappeared.

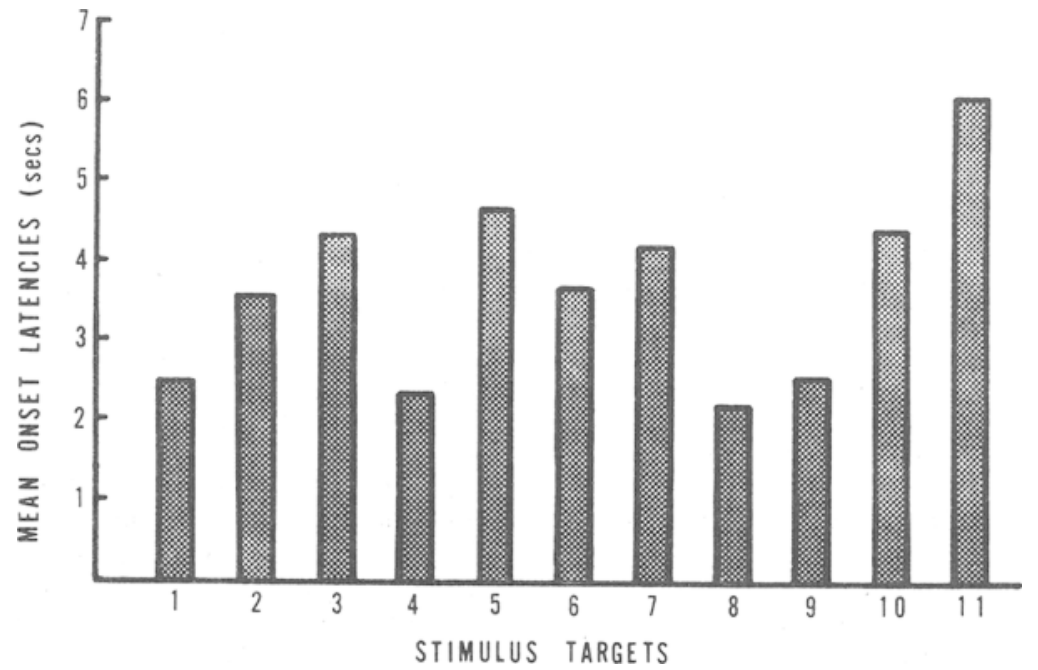

Fig. 4. Mean onset latencies for the 11 stimulus targets.

The Ss reported that the vertical targets, Nos. 1, 2, and 3, showed a "sharpening" effect at the ends before fading. Enclosed targets, such as Nos. 4-9 and 11, underwent a "filling in," which obliterated the outlines of the object (e.g., Nos. 7 and 8). All Ss reported reversal of Target 7 , the Necker cube; in addition; Ss reported that the cube lost its three-dimensional qualities during fading sequences. Target 9 , the triangle and circle, showed alternations in which the triangle would disappear, leaving the circle and vice versa; also, adjacent portions were reported to move closer together.

The results of the two time measures are shown in Figs. 4 and 5. The graphed mean onset latencies for the stimulus targets did not show differentiation when stimulus dimensions, such as thickness, complexity, and area of transmitted light from the target, were independently considered. Figure 5 graphically represents the cumulative times for stimulus targets

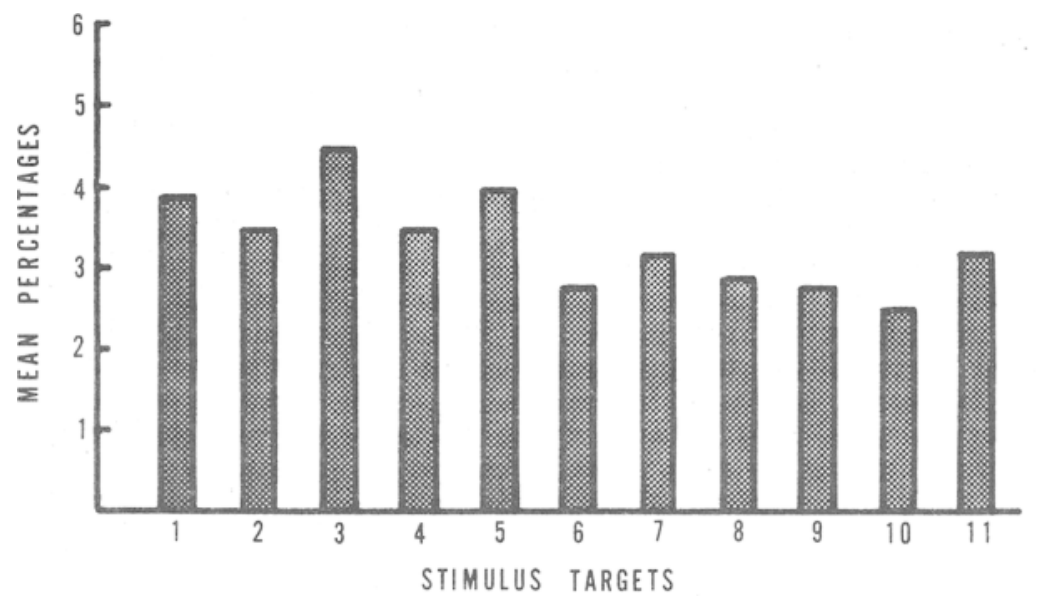

Fig. 5. Mean percentage times targets seen as complete for the trial period. viewed as complete, expressed in terms of percentages. The complete targets were visible for a little more than $3 \%$ of the trial time, regardless of thickness, complexity, and area of transmitted light.

DISCLSSION

The results of fading and image breakdown for flash stabilization compare quite similarly with the findings of corneal type stabilization procedures (e.g.. Pritchard et al, 1960. 1961: Hebb, 1963) and the afterimage method (Bennet-Clark \& Evans, 1963: Evans \& Marsden, 1966: Evans, 1967). The regeneration of stimulus targets during the trial period is a finding at odds with Yarbus's contention that regeneration and breakdown phenomena in corneal systems are the result of faulty stabilization. However, no restraints on bringing back the faded afterimage were put on the Ss; they quickly learned to facilitate bringing back the faded image by blinking, squinting, or closing of the left eye. The two time measures taken here 
undoubtedly were affected by these uncontrolled factors. The methodology may have been improved by not allowing the $S$ to blink and by utilizing a flickering background to sustain the appearance of the afterimage. However, the stroboscopic effect in low light illumination could influence brain rhythm activity and direct the course of fading or regeneration sequences. Some criticism may also be leveled at the way information was elicited from the $S$, i.e., leverpressing and/or verbal accounts. Experimenters disagree on the weight to be accorded verbal responses in lieu of the S's fallible memory. But leverpressing also relies on the same fallible memory in instructions to the $\mathrm{S}$ before the test trials. It was felt that information might be gained by verbal reports without unduly forcing the $S$ to categorize what he thinks is required of him by the $E$.

\section{REFERENCES}

BARLOW, H. B. Slippage of contact lenses and other artifacts in relation to fading and regeneration of supposedly stable retinal images. Quarterly Journal of Experimental Psychology. 1963, 15, 36-51.

BARLOW. H. B., \& SPARROCK, J. M. B. The role of afterimages in dark adaptation. Science, 1964, 144, 1309-1314.

BENNETCLARK, H. C., \& EVANS, C. R. Fragmentation of patterned targets when viewed as prolonged after-images. Nature, 1963,199.1215-1216.

DITCHBURN, R. W., \& FENDER, D. H. The stabilized retinal image. Optica Acta, 1955, 2, 128-133.

DITCHBURN, R. W., \& PRITCHARD, R. M. Vision with a stabilized retinal image. Nature, 1952, 170, 36-38.

EAGLE, M., BOWLING, L., \& KLEIN, G. S. Fragmentation phenomena in luminous designs. Perceptual \& Motor Skills, 1966, 23, 143-152.
I.VANS, C. R., \& PIGGiNS, D. J. A comparison of the behaviour of geometrical hapes when viewed under conditions of steady fixation, and with apparatus for producing a stabilised retinal image. British Journal of Physiological Optucs, 1963, 20, 1-13.

IVANS, C. R., \& MARSDFN, R. P. A study of the effect of perfect retinal stabilization on some well-known visual illusions, using the after-image as a method of compensating for eye movements. British Journal of Physiological Optics, 1966, 23, 242-248.

EVANS, C. R. Further studies of pattern perception and a stabilized retinal image: The use of prolonged afterimages to achieve perfect stabilization. British Journal of Psychology, $1967,58,315-327$.

HEBB, D. O. The semiautonomous process: Its nature and nurture. American Psychologist, $1963,18,16.27$.

HECKENMUELLER, E. G. Stabilization of the retinal image: A review of methods, effects, and theory. Psychological Bulletin, 1965,63, 157-169.

MCKINNEY, J. P. Disappearance of luminous designs. Science, 1963, 140, 403-404.

MINARD, J. G., \& BATCHER, J. Disappearance of naturally fixated luminous stimuli and stability of phase sequences: A selective review and related experiment. Perceptual \& Motor Skills, 1967, 24, 747-752.

PRITCHARD, R. M. Stabilized images on the retina. Scientific American, 1961, 204, $72-78$.

PRITCHARD, R. M., HERON, W., \& HEBB, D. $O$. Visual perception approached by the method of stabilized images. Canadian Journal of Psychology, 1960, 14, 67.77.

RATLIFF, F. The role of physiological nystagmus in monocular acuity. Journal of Experimental Psychology, 1952, 43, 163-172. RIGGS, L. A., RATLIFF, F., CORNSWEET, J. C., \& CORNSWEET, T. N. The disappearance of steadily fixated visual test objects. Joumal of the Optical Society of America, 1953, 43, 495-501.

SCHUCK, J. R., BROCK, T. C., \& BECKER, $L$. A. Luminous figures: Factors affecting the reporting of disappearances. Science, 1964, $146,1598-1599$.

YARBUS, A. L. Eye movement and vision. New York: Plenum Press, 1967.

\section{What is vividness?*}

\section{ELIZABETH P. KIRCHNER \\ The Pennsylvania State University, University Park, Pa. 16802}

The vividness of verbal stimuli is highly related to the semantic differential rating of active (and, in decreasing order of magnitude, to ratings of fast, aggressive, new, hard, and beautiful). Studies manipulating vividness may thus involve demonstration of the effects of the activity dimension on verbal learning.

Vividness $(V)$, a variable of venerable age in theory (Brown, 1854), has been operationally defined in recent work as an intrinsic dimension of verbal stimuli, and its impact upon the learning of verbal materials has been reported (Kirchner, 1969, 1970; Tulving, McNulty, \& Ozier,

*This study was supported by the Central Fund for Research of The Pennsylvania State University.
1965). However, the relationship of $V$ to other variables of contemporary interest has not been delineated.

The present study explored the relationships of rated $V$ with several of the familiar semantic-differential dimensions of Osgood, Suci, \& Tannenbaum (1957), with frequency of occurrence (Thorndike \& Lorge, 1944), and with associative productivity $(m$, Noble, 1952). In previous work (Kirchner, 1969, 1970), the present author hypothesized that $\mathrm{V}$ might be related to the activity and potency dimensions (Osgood et al, 1957) and unrelated to the evaluative dimension. Although considerable work has been directed toward studying the effect of evaluative factors on associative behavior (cf. Cramer, 1968, for an extensive review of this subject), much less effort has been directed toward that of other semantic dimensions, although Wimer (1963) presents data relating some of these dimensions and $m$, and Carroll (1965) offered speculations regarding the psycholinguistic significance of several dimensions.

\section{METHOD}

Selection of words for inclusion in this study was based upon the intent to sample words representative of: (1) the Thorndike \& Lorge (1944) frequency range (counts varying from 1 per million to over 100 per million); (2) flat and steep response hierarchy (selected from the norms of Palermo \& Jenkins, 1964, and of Bousfield, Cohen, Whitmarsh, \& Kincaid, 1961); (3) concrete and abstract nouns (Mednick, Mednick, \& Jung, 1964); and (4) semantic space (Jenkins, Russell, \& Suci, 1958). Also included were words judged to be vivid and dull by the author. The word list was thus designed to encompass the ranges of the variables of interest rather than to consist of randomly selected words.

\section{PROCEDURE}

\section{SEMANTIC RATINGS}

In a procedure following that of Jenkins et al (1958), each of the 62 words utilized in this study was rated on a 7-point scale by $30 \mathrm{Ss}$ (in groups of 20 to $40 \mathrm{Ss}$, approximately half male, half female). Each of the total of 93 Ss rated 20 words, the order of word presentation being randomized. Thirteen scales were used in the rating procedure: nine scales were selected from Jenkins et al (1958); three scales (moral-immoral, wrong-right, and abstract-concrete), for which data are not reported here, were included for purposes extraneous to those of the present study; and one scale (aggressive-nonaggressive) was included because of its hypothesized relationship with V (Gofstein, 1956). The 10 scales to be reported here are listed below.

(1) Vivid-colorless.

(2) Good-bad. This is the pivotal scale for the first semantic differential factor of evaluation, having a loading of 1.00 on that factor.

(3) Hard-soft. This is the pivotal scale (with a loading of .97) for the second semantic differential factor, potency. 